\title{
Criptorquidia: desde la embriología al tratamiento
}

\author{
Sigrid Blanco * \\ Silvia Gottlieb ** \\ Romina Grinspon *** \\ Rodolfo Rey ${ }^{* * *}$
}

*Médico. Especialista en Endocrinología Pediátrica. Bienestar IPS y Salud Total. Valledupar. Cesar. Colombia.

**Médico. Especialista en Endocrinología Pediátrica. Centro de Investigaciones Endocrinológicas "Dr. César Bergadá". CONICET-FEI-División de Endocrinología. Hospital de Niños R. Gutiérrez. Buenos Aires. Argentina.

***Médico. Especialista en Endocrinología Pediátrica. Centro de Investigaciones Endocrinológicas "Dr. César Bergadá". CONICET-FEI-División de Endocrinología. Hospital de Niños R. Gutiérrez. Buenos Aires. Argentina.

**** Médico. Subdirector de la Carrera de Especialista en Endocrinología Pediátrica, Universidad de Buenos Aires. Director del Centro de Investigaciones Endocrinológicas “Dr. César Bergadá". CONICET-FEI-División de Endocrinología, Hospital de Niños R. Gutiérrez. Buenos Aires. Argentina.

Correspondencia: Dra. Sigrid Blanco. Valledupar. Colombia. Correo electrónico: sigridbb@gmail.com

\section{RESUMEN}

La criptorquidia es la anomalía genital más común en el recién nacido varón y a pesar de que su evaluación y tratamiento han progresado con las décadas, siguen existiendo muchas controversias al respecto. En todo niño, el examen físico genital debe buscar la presencia de las gónadas en el escroto, en su ausencia, debe tratar de distinguirse si la anomalía es unilateral o bilateral, definiéndose con la mayor precisión posible la posición de estas y distinguiéndose entre testículo criptórquido, ectópico y retráctil junto con la valoración de la existencia de tejido testicular funcional a través de estudios hormonales. El tratamiento puede ser hormonal o quirúrgico, este último no se recomienda antes del año de edad además corresponde a la terapia más exitosa para reubicar el testículo en el escroto en aquellos pacientes con gónadas en posición inguinal alta, abdominal o en posición ectópica, o en aquellos en los que la terapia hormonal ha fallado. Por otro lado, la terapia hormonal se recomienda en mayor medida cuando las gónadas están en posición inguinal media, baja o escrotal alta. El tratamiento apunta a reducir, aunque no siempre logra evitar los posibles problemas a largo plazo de infertilidad y cáncer de testículo. MÉD UIS. 2015;28(3):371-80.

Palabras clave: Fertilidad. Neoplasia Testicular. Orquidopexia.

\section{Cryptorchidism: from embryology to treatment}

\section{ABSTRACT}

Cryptorchidism is the most frequent genital anomaly in the newborn male. Although significant progress has been made in the assessment and treatment of cryptorchidism, several controversies still exist. In every boy, the physical examination should seek the presence of the gonads in the scrotum. Otherwise, it should be made clear whether cryptorchidism is unilateral or bilateral, the position of the gonad should be defined as precisely as possible, distinguishing between cryptorchidism, ectopia and retractile testes. Hormonal laboratory studies help assessing the existence of functional testicular tissue. Treatment may be hormonal or surgical. The latter is not recommended before the age of 1 year. Surgery has the highest rates of success in patients with gonads in high inguinal, abdominal or ectopic position, or when hormonal treatment has failed. Hormonal treatment may be successful in patients with gonads in low inguinal or high scrotal position. Treatment aims to reduce, although it does not always avoids, the risks of infertility and of testicular cancer in the long term. MÉD UIS. 2015;28(3):371-80.

Keywords: Cryptorchidism. Fertility. Testicular Neoplasm. 
¿Cómo citar este artículo?: Blanco S, Gottlieb S, Grinspon R, Rey R. Criptorquidia: desde la embriología al tratamiento. MÉD UIS. 2015;28(3):371-80.

\section{INTRODUCCIÓN}

La criptorquidia es la anomalía genital más común en el recién nacido, esta es definida como la ausencia del testículo en su posición escrotal normal, aunque la gónada sea palpable y no esté oculta. Algunos autores utilizan el término criptorquidia exclusivamente para referirse al descenso incompleto del testículo, con ubicación dentro del trayecto normal hacia el escroto y distinguen a la ectopia testicular y al testículo

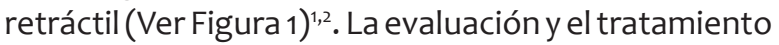
de la criptorquidia han progresado durante décadas pasadas. Sin embargo, a pesar de que esta condición ha sido extensamente estudiada, los conocimientos sobre sus causas y su fisiopatología aún siguen siendo limitados. El término ectopia testicular se ha reservado para referirse a una posición anormal del testículo fuera del trayecto habitual del descenso hacia el escroto, pudiendo estar localizado en región femoral, perineal, abdominal o transversa ; mientras que el testículo retráctil se caracteriza por no estar en el fondo del escroto, pero puede ser llevado a dicha posición sin maniobras dolorosas y permanecer en posición normal luego de la manipulación; no debe ser considerado retráctil cuando este no permanece en el fondo del escroto.

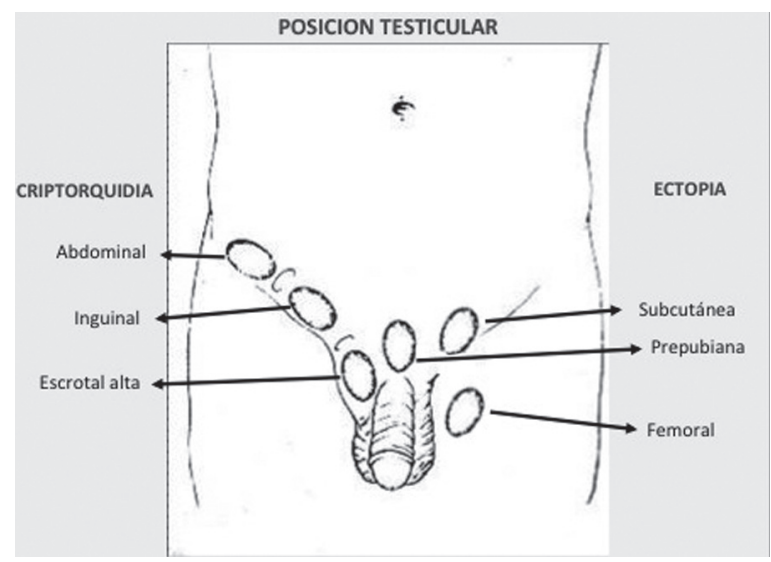

Figura 1. POSICIÓN TESTICULAR. Los testículos mal descendidos pueden hallarse en el camino normal del descenso, llamándose criptórquidos, o bien encontrarse fuera de dicho camino normal, llamándose ectópicos

Fuente: Martínez Mora J. Textbook of intersexual states. Barcelona: Ediciones Doyma, 1994.
Desde un punto de vista clínico, es importante distinguir si el testículo es palpable o no, determinar la altura del testículo según el trayecto inguinal, si la criptorquidia es uni o bilateral, si es aislada o forma parte de un síndrome polimalformativo. Asimismo, la criptorquidia puede ser congénita o adquirida, en este último caso el testículo estuvo presente en el escroto y luego ascendió para ubicarse de modo permanente en posición anómala3. Además, su enfoque diagnóstico y de tratamiento siguen generando controversias.

De acuerdo a diferentes estudios publicados se ha encontrado que la criptorquidia afecta entre 1,6\% y $5,7 \%$ de los recién nacidos a término ${ }^{1,4}$, con una incidencia mayor del 30 a $40 \%$ en recién nacidos pretérmino ${ }^{2,5}$. Los testículos pueden descender al escroto espontáneamente durante el primer año de vida, generalmente en los tres primeros meses de vida en el $75 \%$ de los recién nacidos a término y en el $90 \%$ de los recién nacidos prematuros; así, la prevalencia de criptorquidia disminuye entre $0,8 \%$ y $1,2 \%$ en el primer año de vida.

El riesgo de testículos no descendidos es diez veces mayor en los gemelos de sexo masculino si está presente en uno de ellos, 3,5 veces más alto en los varones que tienen un hermano con testículos no descendidos y 2,3 veces mayor en los hombres cuyo padre hubieran padecido la condición ${ }^{6}$. Entre $1 \%$ y $3 \%$ de testículos previamente descendidos podrían ascender a una situación de criptorquidia ${ }^{7,8}$, observándose una prevalencia entre $0,8 \%$ y 2,5\% a los tres años de vida y hasta $2,6 \%$ a los seis años ${ }^{5}$. Dada la frecuencia del problema, el conocimiento sobre el tema es particularmente importante para los profesionales de la salud. El objetivo de la presente revisión es evidenciar las características del descenso testicular normal y aspectos de la etiología, fisiopatología, clínica, tratamiento y pronóstico en el paciente con criptorquidia.

La revisión bibliográfica se realizó entre junio y diciembre de 2014 usando Pubmed. Se incluyeron los artículos publicados entre 1970 y 2014. Las palabras clave usadas fueron: cryptorchidism, retractile testes, testicular ectopia, maldescended testes. 


\section{DesarRollo y descenso testicular}

Las células germinales primordiales migran desde el saco vitelino hacia las gónadas en desarrollo entre la tercera y quinta semana de vida embrionaria, donde se colonizan los túbulos seminíferos a partir de la sexta semana y dan origen a los gonocitos, que se dividen por mitosis y luego se diferencian en espermatogonias, las cuales son utilizadas como reserva de células espermatogénicas ${ }^{9}$.

El testículo se desarrolla inicialmente en la cavidad abdominal a partir de la cresta gonadal desde la sexta semana de vida embrionaria (octava semana de amenorrea). Las células de Sertoli se desarrollan y forman los cordones testiculares, futuros túbulos seminíferos, y las células de Leydig se ubican en el tejido intersticial ${ }^{10}$. Las células de Sertoli secretan la Hormona Anti-Mülleriana (AMH), que provoca la regresión de los conductos de Müller. En su defecto, dichos conductos dan origen a las trompas de Falopio, el útero y la porción superior de la vagina. Por otro lado, las células de Leydig secretan testosterona que, al unirse al receptor de andrógenos presente en los conductos de Wolff, provoca la diferenciación de estos en epidídimos, conductos deferentes y vesículas seminales. Por acción de la enzima $5 a$-reductasa, la testosterona es transformada en dihidrotestosterona, que se une al receptor de andrógenos en el seno urogenital y en los esbozos de los genitales externos, dando origen a la próstata y la uretra masculina, y se virilizan los genitales externos ${ }^{10}$. Todos estos procesos tienen lugar en las primeras 12 y 13 semanas del desarrollo fetal (Ver Figura 2). En la segunda mitad de la gestación, los andrógenos junto con el Factor Insulino-símil 3 (INSL3), también secretado por las células de Leydig en respuesta a la Gonadotrofina Coriónica Humana (hCG) y luego a la Hormona Luteinizante (LH), intervienen en el descenso del testículo ${ }^{4}$ y su posición definitiva en el escroto (Ver Figura 3).

En la primera fase denominada transabdominal, el testículo está anclado al orificio inguinal interno por medio del gubernaculum testis. El factor INSL3, liberado por las células de Leydig, se une a su receptor LGR8 o RFXP2, que se encuentra presente en el gubernaculum, lo cual genera un aumento de tamaño del mismo. A su vez, el ligamento suspensorio craneal, que une al testículo con la pared abdominal posterior, entra en regresión por acción de la testosterona. Así, el testículo y epidídimo rodeados de peritoneo se deslizan e ingresan en el conducto inguinal hacia la semana 15 de vida fetal"1.

En la segunda fase o ínguinoescrotal, el testículo desciende a través del conducto inguinal hasta su posición definitiva en el fondo escrotal, guiado por el gubernaculum testis. Este proceso es altamente dependiente de andrógenos, aunque también intervienen otros factores como el CGPR y HOXA10 y el aumento de la presión intraabdominal ${ }^{11-13}$. Esta fase se completa en más del $90 \%$ de los fetos hacia la semana 35 de gestación ${ }^{12}$. Una vez que el testículo se ha localizado en la base del escroto, el gubernaculum se fibrosa y la conexión peritoneal se cierra.

\section{DIFERENCIACION GENITAL MASCULINA}

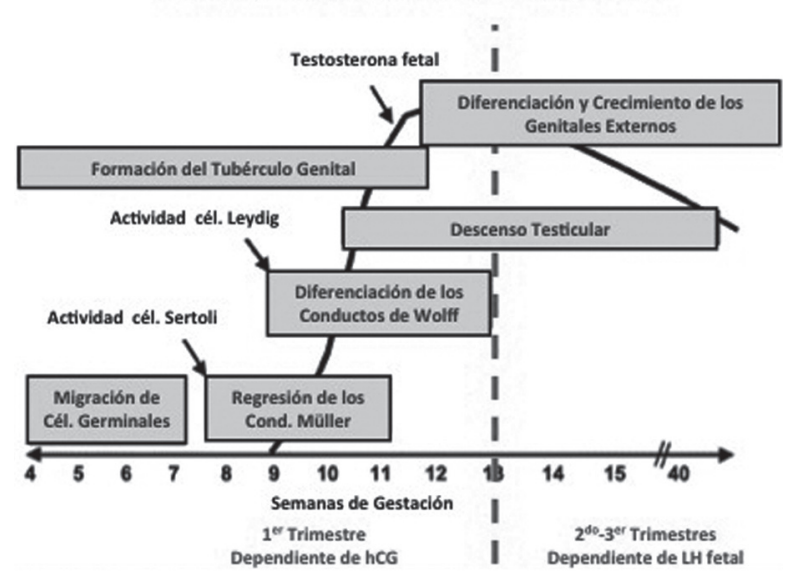

Figura 2. ETAPAS DE LA DIFERENCIACIÓN SEXUAL FETAL MASCULINA. En el 10 trimestre ocurre la diferenciación gonadal. Por acción de la hCG placentaria, la células de Leydig secretan testosterona que provoca la masculinización de los conductos de Wolff, del seno urogenital y de los genitales externos. En la $2^{a}$ mitad de la gestación, la LH se transforma en el estímulo principal de las células de Leydig. En esta etapa, los genitales ya virilizados (próstata, pene, escroto) aumentan de tamaño y los testículos completan su descenso. Modificada con permiso de: Klonisch T, Fowler PA, Hombach-Klonisch S. Molecular and genetic regulation of testis descent and external genitalia development. Developmental Biology, 270: 1-18, 2004. Copyright: Elsevier 2004.

Tras el nacimiento, el volumen testicular se incrementa levemente, pero dicho incremento es imperceptible clínicamente cuando se compara con el orquidómetro de Prader (Ver Figura 4). El volumen testicular durante la infancia está representado esencialmente por las células de Sertoli, mientras los otros elementos testiculares son minoritarios. En la edad puberal, el testículo aumenta notoriamente de tamaño, debido a la proliferación y diferenciación de las células germinales conformando la espermatogénesis puberal y adulta (Ver Figura 5). 


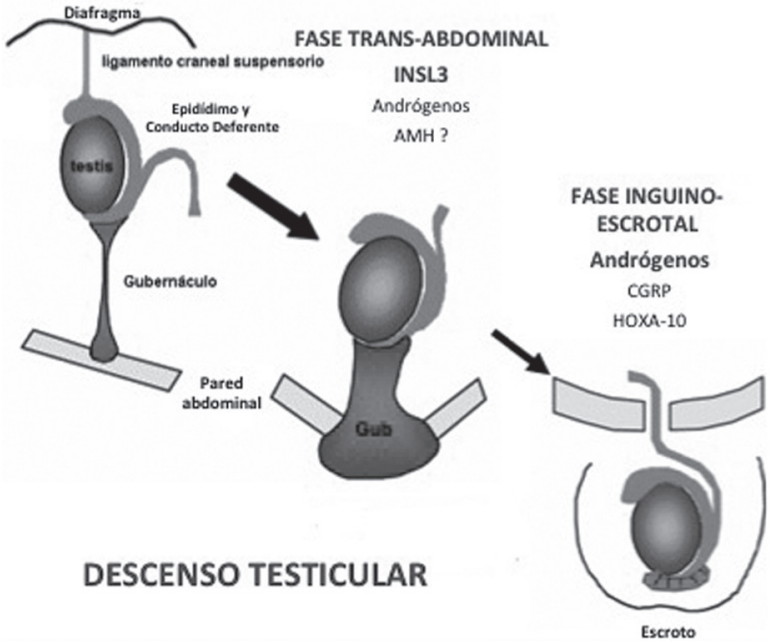

Figura 3. DESCENSO TESTICULAR. Los testículos se forman en las crestas gonadales en posición abdominal. Están ligados a la pared abdominal póstero-superior mediante el ligamento craneal y a la pared inferior mediante el gubernáculo (o gubernaculum testis). Hay 2 fases en el descenso testicular: la primera, trans-abdominal, depende esencialmente del Factor Insulino-símil 3 (INSL3) y de los andrógenos, en tanto que la segunda, ínguino-escrotal, depende principalmente de los andrógenos. Otros factores como el Péptido Relacionado con la Calcitonina (CGRP) y el Factor Homético HOXA10 también juegan un rol. Modificada con permiso de: Klonisch T, Fowler PA, Hombach-Klonisch S. Molecular and genetic regulation of testis descent and external genitalia development. Developmental Biology, 270: 1-18, 2004. Copyright: Elsevier 2004.

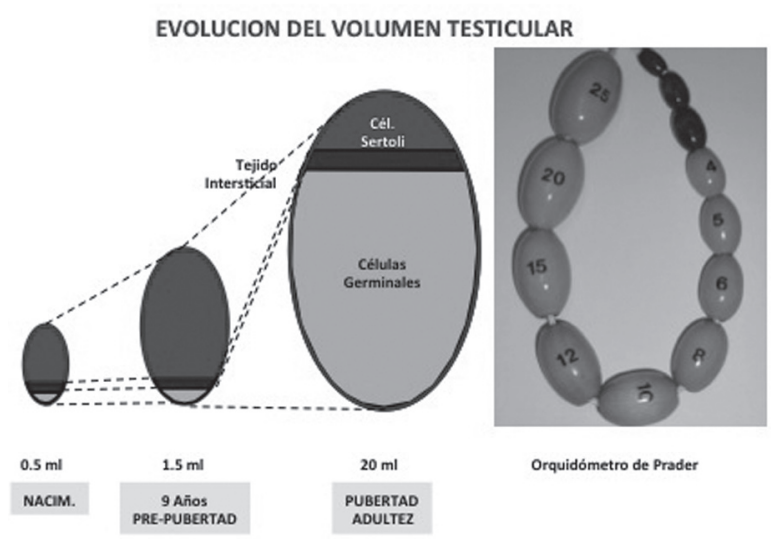

Figura 4. EVOLUCIÓN DEL VOLUMEN TESTICULAR. Los testículos tienen un volumen testicular de aproximadamente $0,5 \mathrm{ml}$ al nacimiento. Durante la infancia, el volumen crece hasta aproximadamente $1,5 \mathrm{ml}$. Dicho crecimiento se debe sobre todo a la multiplicación de las células de Sertoli. En la pubertad, hay un incremento notorio del volumen testicular, debido esencialmente a la proliferación de las células germinales que desarrollan la espermatogénesis completa (es decir mitosis de espermatogonias, meiosis y espermiogénesis, ver Fig. 5). Clínicamente el volumen testicular se evalúa por comparación con el orquidómetro de Prader (derecha). Esta medición es menos precisa, ya que involucra también al epidídimo y las membranas que rodean al testículo: por ello, el volumen testicular es considerado prepuberal hasta los $3 \mathrm{ml}$ y puberal a partir de los $4 \mathrm{ml}$. Modificado con permiso de: Rey R. Regulation of spermatogenesis. Endocr Dev 2003; 5:38-55. Copyright: Karger 2003

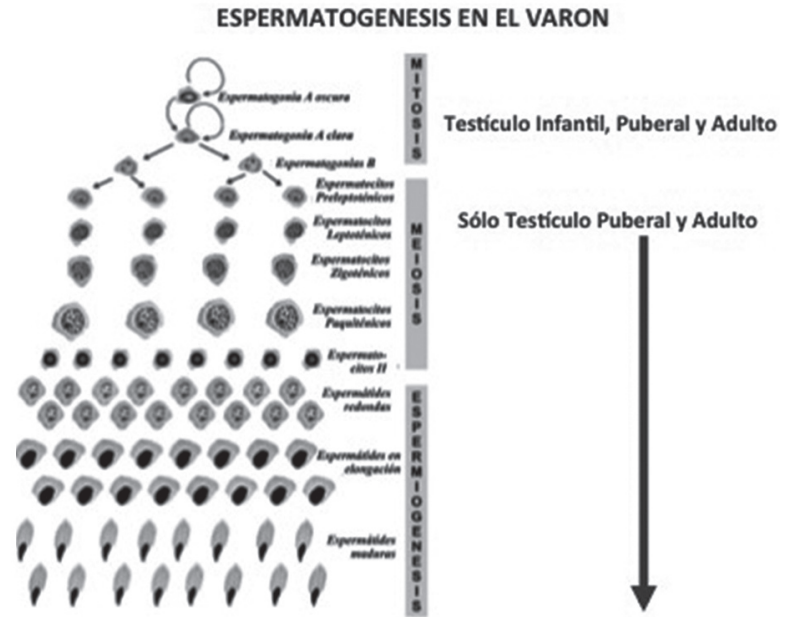

Figura 5. ESPERMATOGÉNESIS EN EL VARÓN. En las etapas fetal e infantil, la espermatogénesis se limita a la proliferación por mitosis de las células germinales más indiferenciadas (células germinales primordiales, gonocitos y espermatogonias). A partir de la pubertad, la espermatogénesis avanza comenzando la meiosis y la espermiogénesis, que dará lugar a la formación de espermatozoides. Modificada con permiso de: Rey R. Regulation of spermatogenesis. Endocr Dev 2003; 5:38-55. Copyright: Karger 2003.

\section{Etiopatiogenia}

La criptorquidia no es una entidad nosológica sino un signo que puede estar presente en el cuadro clínico de diferentes entidades. En gran parte de los casos, la criptorquidia se presenta como manifestación única dentro del cuadro clínico del paciente y su etiología y patogenia son desconocidas; se postula que factores genéticos hormonales, ambientales y anatómicos pueden estar involucrados. La evidencia que apoya la susceptibilidad genética en los niños con criptorquidia es actualmente débil, aunque se han implicado varios genes incluyendo INSL3, RFXP2, $A R$, ESR1 y $A M H^{7,8}$.

A su vez, varios factores hormonales, tales como: la LH, la hCG y los andrógenos juegan papeles importantes en el mecanismo de descenso testicular en el escroto, algunos autores, también proponen que la $\mathrm{AMH}$ podría tener efecto en este proceso, aunque esto es todavía un tema controvertido ${ }^{11,14}$. El bajo nivel de estrógenos maternos y la insuficiencia placentaria con disminución de la secreción de hCG también son sugeridas como factores de riesgo de testículos no descendidos². Asimismo, la mayor prevalencia de testículos no descendidos en los casos con síndrome de Prader-Willi, síndrome de Kallman, hipoplasia pituitaria y la anencefalia indica el papel crítico del eje hipotálamo-hipofisario. La exposición a factores ambientales que funcionan como disruptores endocrinos, como los compuestos 
organoclorados, los mono-ésteres de los ftalatos y el tabaquismo materno que pueden ser factores de riesgo ${ }^{2}$.

Por otro lado, para su normal funcionamiento, la gónada masculina debe ubicarse en el escroto, donde la temperatura es aproximadamente de 1 a $3^{\circ} \mathrm{C}$ inferior a la temperatura corporal central. La posición anómala del testículo en la cavidad abdominal o el conducto inguinal expone a las poblaciones celulares de la gónada a temperaturas superiores a las requeridas para su función normal. Modelos animales de experimentación han permitido elucidar que el aumento de la temperatura por criptorquidia experimental afecta esencialmente a los espermatocitos meióticos y las espermátides, las cuales degeneran por una apoptosis acelerada. Es más discutido el efecto de la temperatura sobre las espermatogonias ${ }^{15}$; trabajos recientes demuestran la existencia de un período crítico en los primeros meses de la vida, durante los que se produce una pérdida de espermatogonias en la gónada mal descendida ${ }^{14,16}$.

\section{Fisiopatología}

En pacientes criptórquidos puede desarrollarse un hipogonadismo primario o testicular, en donde se presenta una función defectuosa de los testículos, siendo la falla gonadal la causa primaria. Cuando todo el tejido testicular está afectado, las células de Leydig producen escasos niveles de testosterona e INSL3, las células de Sertoli secretan escasa AMH y las células germinales están muy disminuidas o ausentes. $\mathrm{Si}$ es de establecimiento fetal temprano, primer trimestre, la deficiencia androgénica y de INSL3 lleva a criptorquidia y ambigüedad genital de severidad variable, con restos müllerianos por deficiencia de $\mathrm{AMH}$. Si el defecto es de establecimiento fetal tardío, segundo o tercer trimestre, los genitales internos y externos son masculinos pero las gónadas no están en el escroto y suele haber micropene de grado variable ${ }^{17}$ (Ver Figura 3). En el hipogonadismo secundario, central o hipotálamo-hipofisario, cuando la afección compromete al eje hipotálamo-hipofisario y a las gónadas simultáneamente, la disfunción gonadal es consecuencia de una deficiencia de las gonadotrofinas. En este caso, también puede tratarse de un hipogonadismo global y manifestarse por criptorquidia, microorquidismo o micropene $e^{17,18}$. Existen formas duales de hipogonadismo, en las que el eje hipotálamo-hipofisario y la gónada están afectadas independientemente. Ejemplos de hipogonadismo dual son el síndrome de Prader-Willi' ${ }^{19}$ y ciertas mutaciones del gen $D A X^{120}$; en este último caso coexiste junto con falla suprarrenal ya que $D A X_{1}$ interviene independientemente en el desarrollo de la hipófisis, del testículo y de las glándulas suprarrenales.

\section{Evaluación clínICA}

La evaluación del paciente con criptorquidia difiere según la edad. En el recién nacido la anamnesis puede aportar datos de interés como la edad gestacional $y$ el peso al nacimiento del neonato, el origen étnico, antecedentes familiares de criptorquidia o hipogonadismo hipogonadotrófico. Un diagnóstico diferencial de importancia en recién nacidos sin gónadas palpables es la hiperplasia suprarrenal congénita 46,XX con virilización completa. Por otra parte, otro diagnóstico relevante en el recién nacido que se presenta como un cuadro de escroto agudo es la torsión testicular perinatal relacionada con un traumatismo periparto, capaz de provocar una atrofia total del tejido testicular, siendo el diagnóstico diferencial el hematoma escrotal sin torsión testicular ${ }^{21}$.

Al examen físico, la evaluación del peso y la longitud corporal del recién nacido son relevantes. La longitud del pene por debajo de los valores de referencia, asociado a criptorquidia, sugiere un posible hipogonadismo primario o secundario. Asimismo, las características escrotales son de suma importancia ya que la existencia de escroto plano y poco rugoso es indicio de que el testículo nunca estuvo en posición normal. Malformaciones de la línea media, como labio y paladar hendidos, pueden orientar el diagnóstico hacia un hipogonadismo central o hipotálamohipofisario ${ }^{22,23}$. La criptorquidia asociada a micropene puede ser indicio de hipogonadismo central, aún en ausencia de malformaciones de la línea media. Además, la debilidad de la pared abdominal anterior sugiere una falla del descenso testicular debida a una insuficiente presión intraabdominal conocida como síndrome del abdomen en ciruela pasa o prune belly ${ }^{24}$.

En todo recién nacido sin gónadas palpables, para descartar el diagnóstico de hiperplasia suprarrenal congénita $46, X X$, es recomendable realizar un iongrama, cariotipo y dosaje de $17-\mathrm{OH}$-progesterona, un valor elevado de esta última confirma el diagnóstico, con lo cual se puede concluir que las técnicas de laboratorio conforman una importante ayuda diagnóstica. Igualmente, el cariotipo puede ser de ayuda para descartar un síndrome de Klinefelter 
47,XXY o sus variantes. En varones criptórquidos la frecuencia de síndrome de Klinefelter es de aproximadamente $1 \%$, o sea casi 10 veces mayor que en varones no criptórquidos ${ }^{25,26}$. La existencia de niveles de testosterona, INSL3, AMH e inhibina $B$ detectables permite descartar una anorquia; se conoce que el INSL3 se encuentra disminuido en pacientes criptórquidos independientemente de la severidad de la criptorquidia ${ }^{27}$. La AMH es altamente confiable para confirmar la presencia de tejido testicular ${ }^{28,29}$ y puede estar baja en los casos de criptorquidia unilateral o bilateral por hipogonadismo primario o secundario. La inhibina $B$ es otro marcador muy confiable de la existencia de tejido testicular ${ }^{30}$. Si la $\mathrm{AMH}$ es no detectable en un paciente criptórquido y la inhibina B es detectable, la sospecha de síndrome de persistencia de conductos de Müller es alta28,29. En cambio, una inhibina $B$ no detectable junto con FSH y LH elevadas, es altamente indicativo de anorquia. Sin embargo, se debe destacar que las gonadotrofinas pueden no estar elevadas, aún en casos de anorquia, entre los seis y los 11 años $^{31}$ (Ver Figura 6). La detección de AMH e inhibina B bajas con gonadotrofinas no elevadas es altamente sugestivo de hipogonadismo secundario ${ }^{32}$. En el síndrome de Klinefelter, las hormonas del eje hipófiso-gonadal están normales en esta etapa de la vida33,34, si bien algunos autores han encontrado niveles ligeramente disminuidos de testosterona ${ }^{35,36}$.
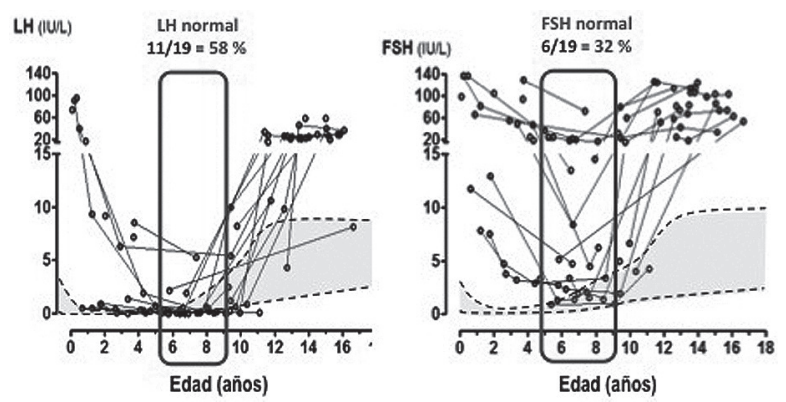

Figura 6. NIVELES SÉRICOS DE GONADOTROFINAS EN VARONES ANÓRQUIDOS. Izquierda: La LH tiene en general valores más bajos que la FSH en varones anórquidos, pudiendo ser normal en más de la mitad de los niños entre los 6 y 9 años de edad. Derecha: La FSH suele mantenerse más elevada, pero puede normalizarse en casi un tercio de los casos entre Iso 6 y 9 años de edad. Modificado con permiso de: Grinspon RP, Ropelato MG, Bedecarrás $P$ et al. Gonadotrophin secretion pattern in anorchid boys from birth to pubertal age: pathophysiological aspects and diagnostic usefulness. Clin Endocrinol (Oxf). 2012; 76:698-705. Copyright: Blackwell Publishing Ltd 2012.

Los métodos de diagnóstico por imágenes son de escasa utilidad en la evaluación de recién nacidos con gónadas no palpables. En cambio, pueden ser útiles ante dudas por la palpación de estructuras compatibles con gónadas en posición inguinal.
La ecografía es la metodología más utilizada en la búsqueda de testículos no descendidos puesto que permite confirmar la existencia de testículos pequeños o rudimentarios presentes en las bolsas con una especificidad del 95\%37-39. Sin embargo, su sensibilidad es baja y puede ser que no llegue a detectar gónadas en conducto inguinal o en cavidad abdominal43,44. El valor predictivo de una ecografía negativa es poco satisfactorio, con hasta un $84 \%$ de falsos negativos según las series ${ }^{44}$. Adicionalmente la tomografía computada y la resonancia magnética nuclear tampoco tienen sensibilidad suficiente ${ }^{40}$, su única utilidad es la capacidad para definir el tamaño de los testículos cuando son detectados.

En niños de uno a seis meses la evaluación no difiere demasiado de la descrita para el neonato. El diagnóstico de hiperplasia suprarrenal congénita en pacientes 46,XX completamente virilizadas sin gónadas palpables es menos probable a esta edad. Por otro lado, en niños mayores de seis meses, es importante indagar si hubo gónadas palpables en algún momento para descartar una criptorquidia adquirida5,6. La evaluación de la progresión pondoestatural, la búsqueda de anosmia y la medición del pene puede orientar ante la sospecha de insuficiencia hipofisaria, mientras que las características del escroto pueden sugerir si existieron gónadas en posición eutópica o no. Los niveles basales de FSH, $\mathrm{LH}$ y testosterona disminuyen notoriamente en el segundo semestre de la vida, siendo de poca ayuda los valores bajos, ya que no es posible distinguir valores anormalmente bajos en esta edad con los métodos de análisis habituales. Al contrario, la determinación de $\mathrm{AMH}$ tiene alta sensibilidad y especificidad para detectar la presencia de testículos no palpables. Un valor normal de $\mathrm{AMH}$ tiene un valor predictivo del 98\% para la presencia de testículos en varones con genitales externos normales y gónadas no palpables, mientras que una $\mathrm{AMH}$ indetectable tiene un valor predictivo del $92 \%$ para la ausencia de tejido testicular ${ }^{29}$. En el síndrome de Klinefelter, la $\mathrm{AMH}$, la inhibina $\mathrm{B}$ y las gonadotrofinas están normales en esta etapa de la vida34.

Para interpretar los niveles séricos de testosterona durante esta etapa de la infancia es necesario realizar una prueba de hCG, ya que no existen células de Leydig funcionalmente activas. Un protocolo simple y útil con fines diagnósticos es el de dos inyecciones intramusculares de 2500 UI hCG en los días uno y cuatro de la prueba y acompañado de extracción de sangre al día siete: en este caso un valor de 
testosterona superior a $1 \mathrm{ng} / \mathrm{ml}$ es considerado como respuesta positiva ${ }^{41}$. Otro protocolo consiste en una dosis única intramuscular de hCG 5000 Ul/1,7 m² de superficie corporal y valoración de la testosterona 48 a 72 horas más tarde ${ }^{42}$, en la respuesta normal debe superar los $2 \mathrm{ng} / \mathrm{ml}$. Aún así, la testosterona posthCG muestra menor sensibilidad y especificidad que la $\mathrm{AMH}$ en el diagnóstico de anorquia, con un valor predictivo para detectar la presencia de testículos del $83 \%$ y para el diagnóstico de anorquia del $69 \% 29$. La administración de hCG puede realizarse también como prueba terapéutica buscando el descenso testicular $1000 \mathrm{UI} /$ semana durante cinco semanas. En estos casos, aunque la prueba terapéutica no logre el descenso testicular, será posible evaluar si hay gónadas en posición ectópica y su capacidad esteroidogénica realizando un dosaje de testosterona 48 a 72 horas después de la última inyección de hCG.

La laparoscopia es un procedimiento diagnóstico y terapéutico altamente eficaz en pacientes con criptorquidia, sin embargo, se ha demostrado un índice de falsos negativos del $8 \%$ en la detección de tejido testicular ${ }^{43}$. Dado el altísimo valor predictivo para diagnosticar una anorquia que tienen en conjunto los niveles indetectables de $\mathrm{AMH}$ e inhibina $B$, junto con la falta de respuesta a la prueba de hCG y la elevación exagerada de los niveles de FSH, no parecería necesario confirmar la ausencia de gónadas mediante un procedimiento quirúrgico.

En varones en edad puberal la consulta de un paciente con criptorquidia es poco frecuente. Dada la reactivación del eje hipotálamo-hipófiso-gonadal los exámenes hormonales muestran un aumento de la LH, la FSH, inhibina B y los niveles de testosterona junto con una caída de la $\mathrm{AMH}$, en el estadio III de Tanner. La falta de aumento en el tamaño del pene y del desarrollo del vello pubiano son claros indicios de una insuficiente producción de testosterona, hipogonadismo primario o secundario, acompañado de otras características de ausencia de desarrollo puberal como la falta del empuje puberal, retraso de la edad ósea, etc. Sin embargo, la adrenarquia está presente y puede aparecer vello pubiano escaso con genitales no estimulados.

El diagnóstico diferencial entre anorquia e hipogonadismo primario o secundario se plantea en un varón de edad puberal sin gónadas palpables ni signos de desarrollo. El hallazgo de inhibina $B$ y $\mathrm{AMH}$ detectables indican la existencia de tejido testicular, mientras que valores no detectables junto con un aumento exagerado de la FSH son diagnósticos de anorquia. Ante la sospecha de un hipogonadismo hipotálamo-hipofisario, la FSH basal o luego de una infusión de Hormona Liberadora de Gonadotrofinas ( $\mathrm{GnRH}$ ) puede ser de gran utilidad. Un valor de FSH basal menor de 1,2 UI/L confirma un hipogonadismo hipotálamo-hipofisario; si la FSH es mayor a 1,2 UI/L pero el pico de FSH es menor a 4,6 UI/L y el de LH es menor a 5,8 UI/L, el diagnóstico de hipogonadismo hipotálamo-hipofisario es altamente probable ${ }^{44}$. Finalmente, una prueba de hCG con determinación de testosterona o INSL3 permite evaluar la existencia y capacidad funcional de las células de Leydig ${ }^{27,45}$.

En pacientes sin gónadas palpables, o con estructuras compatibles con gónadas palpables en posición escrotal o inguinal, la existencia de signos de desarrollo puberal indican que existe o existió tejido testicular capaz de producir andrógenos. Puede plantearse el diagnóstico de Síndrome de Klinefelter así como también el de una degeneración completa del tejido testicular secundaria a traumatismo, cirugía, torsión, entre otros. La existencia de niveles normales de gonadotrofinas, testosterona, INSL3, $\mathrm{AMH}$ e inhibina $\mathrm{B}$ no descartan el diagnóstico de síndrome de Klinefelter en estadios tempranos del desarrollo puberal34. A partir del estadio de Tanner III, tanto las gonadotrofinas como las hormonas testiculares están anormales en el síndrome de Klinefelter $34,35,46$.

\section{TratAMIENTO}

El objetivo del tratamiento es lograr la localización persistente en el escroto sin atrofia obvia, con una adecuada espermatogénesis y fertilidad, en pro de la prevención del cáncer testicular.

En la práctica clínica, la elección de la terapia se basa en la edad de presentación, en la ubicación y -aspecto del testículo criptórquido. Una conducta expectante se puede mantener en niños menores de un año de edad con testículos con ubicación baja en el canal inguinal, ya que el descenso espontáneo sigue siendo una posibilidad a esta edad. Sin embargo, no parece prudente retrasar innecesariamente el tratamiento, dada la posibilidad de deterioro de las células germinales ${ }^{47,48}$. Claramente, el tratamiento debe realizarse antes del inicio de la pubertad para evitar un daño permanente de los túbulos seminíferos y para disminuir el riesgo de desarrollo tumoral ${ }^{49}$. 


\section{TRATAMIENTOS HORMONALES}

Los tratamientos hormonales más utilizados en pacientes con criptorquidia incluyen la hCG y la $\mathrm{GnRH}$, ya sea en forma separada o combinada. Si bien existen diferentes protocolos de tratamiento con hCG, uno ampliamente difundido utiliza $1000 \mathrm{UI}$ de hCG por vía intramuscular una vez por semana durante cinco semanas, evaluando la posición de las gónadas en la semana que sigue a la última inyección. El tratamiento con $\mathrm{GnRH}$ consiste en la

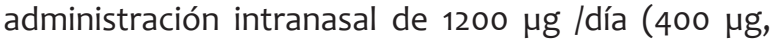
tres veces por día) durante cuatro semanas ${ }^{50}$. La eficacia del tratamiento hormonal es variable según los diferentes estudios, con tasas de éxito que varían entre $0 \%$ y $55 \%$ para la hCG, y entre $9 \%$ y $78 \%$ para la $\mathrm{GnRH}^{51,52}$. La eficacia es mayor cuanto más bajas se encuentren las gónadas antes del tratamiento, sin mostrar demasiada diferencia si se realiza en niños prepuberales antes o después de los cuatro años de edad (Ver Figura 7).

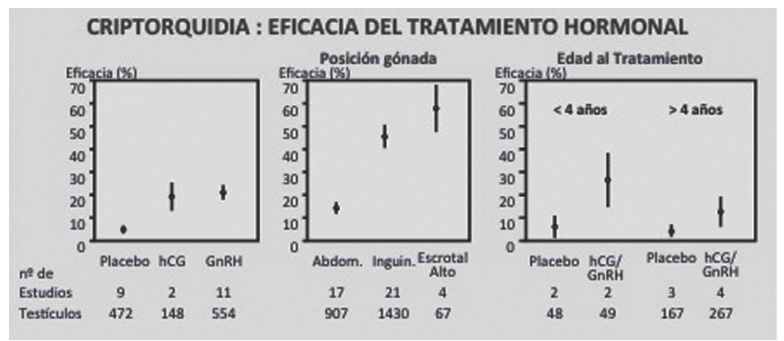

Figura 7. EFICACIA DEL TRATAMIENTO HORMONAL DE LA CRIPTOROUIDIA Izquierda: considerando todos los tipos de criptorquidia, la hCG y la GnRH tienen más eficacia que el placebo para provocar el descenso testicular. Centro: la eficacia del tratamiento hormonal es mayor cuanto más bajas se encuentren las gónadas antes del tratamiento. Derecha: el tratamiento hormonal es apenas más eficaz antes de los 4 años que después de esa edad. Modificado con permiso de: Pyörälä S, Huttunen NP, Uhari M. A review and meta-analysis of hormonal treatment of cryptorchidism. Journal of Clinical Endocrinology and Metabolism, 80: 2795-2799, 1995. Copyright: The Endocrine Society 1995.

\section{TRATAMIENTO QUIRÚRGICO}

El tratamiento quirúrgico de la criptorquidia, incluyendo la ectopia testicular, puede tener dos alternativas: la cirugía a cielo abierto o la laparoscopia. La elección de uno u otro procedimiento depende de la experiencia del equipo quirúrgico. Como ya se ha discutido anteriormente, la laparoscopia puede tener fines diagnósticos y terapéuticos al mismo tiempo ${ }^{53}$. Varios autores proponen iniciar el procedimiento mediante laparoscopia para tratar de ubicar la gónada en el abdomen. Si la gónada no está presente, se sugiere buscar el conducto deferente y los vasos espermáticos; si se encuentran entrando en el conducto inguinal, se procede a la exploración inguinal, mientras si se encuentra una terminación ciega del deferente y de los vasos, se suspende la exploración ya que se concluye que la gónada no existe. Por otro lado, si en la exploración inicial se encuentran gónadas atróficas en el abdomen, se propone la orquidectomía laparoscópica. Si los testículos tienen aspecto normal y el cordón es largo, se sugiere la orquidopexia laparoscópica, mientras que si el cordón es corto, se propone proceder al autotransplante microvascular o la orquidopexia en dos tiempos, Fowler-Stephens. Una revisión sistemática reciente ${ }^{51}$ muestra que el tratamiento quirúrgico se asocia con tasas de éxito de descenso testicular que van desde $33 \%$ a $100 \%$, dependiendo de la cirugía. La tasa de éxito fue del $78,7 \%$ para el procedimiento de Fowler-Stephens en un tiempo, 86\% para Fowler-Stephens en dos tiempos y $96,4 \%$ para orquiodopexia primaria. Las tasas fueron similares entre los estudios que compararon la cirugía laparoscópica y a cielo abierto. Los efectos asociados específicamente con la reparación quirúrgica fueron raros.

\section{Pronóstico}

Dentro de los aspectos más relevantes del pronóstico del paciente con criptorquidia se encuentra el riesgo de cáncer testicular, torsión testicular e infertilidad. Primero, la presencia de criptorquidia está asociada esencialmente con la infertilidad y un aumento del riesgo de neoplasia gonadal.

El riesgo de padecer cáncer testicular es de 35 a 48 veces mayor además de un aumento de la posibilidad de degeneración maligna en los pacientes con testículos no descendidos en comparación con la población general ${ }^{2}$. Varios trabajos concluyeron que el riesgo de malignidad puede reducirse por orquiodopexia temprana ${ }^{2,49}$, aunque otros han discutido dichos resultados ${ }^{54,55}$. De hecho, entre un $10 \%$ y un $25 \%$ de los tumores se observan en el testículo contralateral normalmente ubicado en el escroto ${ }^{56}$, lo cual se explicaría mediante la hipótesis del "síndrome de disgenesia testicular" que asocia criptorquidia, infertilidad y cáncer testicular ${ }^{57}$. El rango de edad durante el cual los tumores testiculares se desarrollan más frecuentemente es entre los 20 y 40 años ${ }^{2}$, siendo los tipos más comunes de cáncer testicular encontrado son el seminoma y carcinoma embrionario ${ }^{2,58}$. 
Segundo, la incidencia de torsión testicular es aproximadamente diez veces mayor en el testículo criptórquido que en el testículo escrotal. El desarrollo de un tumor gonadal también aumenta el riesgo de torsión ${ }^{59}$.

Finalmente, la asociación entre criptorquidia e infertilidad ha sido bien documentada ${ }^{60}$. Encontrándose que el testículo en posición anómala durante la pubertad conduce hacia una falla espermatogénica completa debido a la afectación de la meiosis y la espermiogénesis (Ver Figura 4). Un estudio retrospectivo mostró un 38\% de infertilidad en hombres con criptorquidia bilateral en comparación con un grupo control (6\% infértiles), lo que indica un aumento de seis veces el riesgo de infertilidad en individuos con criptorquidia bilateral. Por el contrario, los hombres con criptorquidia unilateral tuvieron una tasa de paternidad del 89,5\%, que es similar al nivel de fertilidad encontrado en otros estudios de la población general con 94\%7. La biopsia testicular durante la orquidopexia puede ser útil en la predicción de la fertilidad futura en pacientes con criptorquidia (Ver Figura 8); testículos sin espermatogonias tienen mal pronóstico independientemente de la edad de la cirugía ${ }^{61,62}$.

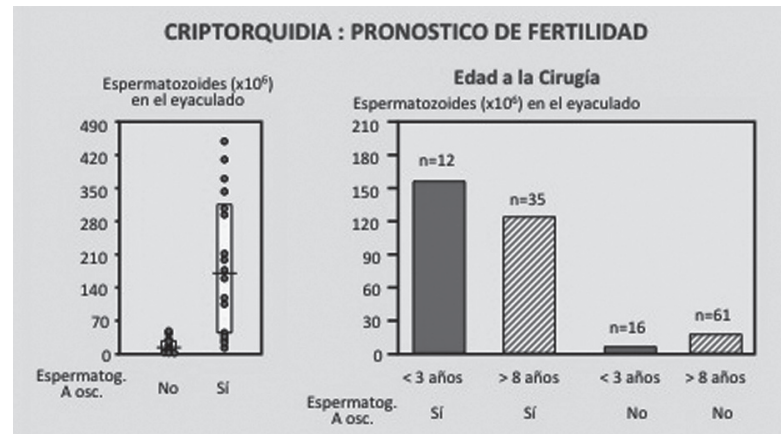

Figura 8. PRONÓSTICO DE FERTILIDAD EN PACIENTES OPERADOS POR CRIPTOROUIDIA. La cantidad de espermatozoides en el eyaculado está relacionada con la existencia de espermatogonias $\mathrm{A}$ en la biopsia al momento de la orquidopexia, pero no con la edad a la que se realizó la misma. Modificado con permiso de: Hadziselimovic F, Herzog B. The importance of both an early orchidopexy and germ cell maturation for fertility. Lancet, 358: 1156-1157, 2001, Copyright: The Lancet Publishing Group 2001, y Hadziselimovic F, Hocht B, Herzog B, Buser MW. Infertility in cryptorchidism is linked to the stage of germ cell development at orchidopexy. Hormone Research, 68: 46-52, 2007, Copyright: Karger 2007.

\section{ConClusiones}

La criptorquidia al ser una de las patologías frecuentes en la población pediátrica debe ser considerada durante la exploración rutinaria del varón recién nacido, el examen físico genital es imprescindible para valorar la presencia de las gónadas. Es importante distinguir con la mayor precisión la posición de las gónadas, si la anomalía es unilateral o bilateral y a su vez diferenciar entre testículos criptórquido, ectópico y retráctil, sin embargo, la resolución quirúrgica no se recomienda antes del año de edad. Por su parte, la terapia hormonal es recomendada sobre todo cuando las gónadas están en posición inguinal media, baja, o escrotal alta.

El reposicionamiento escrotal con éxito de los testículos puede reducir, pero no evitar los posibles problemas a largo plazo de infertilidad y cáncer de testículo. Un asesoramiento adecuado y el seguimiento del paciente a largo plazo son necesarios.

\section{Referencias Biblográficas}

1. Sijstermans K, Hack WW, Meijer RW, van der Voort-Doedens LM. The frequency of undescended testis from birth to adulthood: a review. Int J Androl. 2008; 31:1-11.

2. Abaci A, Catli G, Anik A, Bober E. Epidemiology, classification and management of undescended testes: does medication have value in its treatment? J Clin Res Pediatr Endocrinol. 2013; 5:65-72.

3. Ghirri P, Ciulli C, Vuerich $\mathrm{M}$ et al. Incidence at birth and natural history of cryptorchidism: a study of 10,730 consecutive male infants. J Endocrinol Invest. 2002; 25:709-15.

4. Preiksa RT, Zilaitiene B, Matulevicius V et al. Higher than expected prevalence of congenital cryptorchidism in Lithuania: a study of 1204 boys at birth and 1 year follow-up. Hum Reprod. 2005; 20:1928-32.

5. Agarwal PK, Diaz M, Elder JS. Retractile testis--is it really a normal variant? J Urol. 2006; 175:1496-9.

6. Hack WW, Sijstermans K, van DJ et al. Prevalence of acquired undescended testis in 6-year, 9-year and 13-year-old Dutch schoolboys. Arch Dis Child. 2007; 92:17-20.

7. Lee PA, Houk CP. Cryptorchidism. Curr Opin Endocrinol Diabetes Obes. 2013; 20:210-6.

8. Pastuszak AW, Lipshultz LI. AUA guideline on the diagnosis and treatment of cryptorchidism. J Urol. 2014; 192:346-9.

9. Dym M, He Z, Jiang J et al. Spermatogonial stem cells: unlimited potential. Reprod Fertil Dev. 2009; 21:15-21.

10. Rey RA, Grinspon RP. Normal male sexual differentiation and aetiology of disorders of sex development. Best Pract Res Clin Endocrinol Metab. 2011; 25:221-38.

11. Nation TR, Balic A, Southwell BR et al. The hormonal control of testicular descent. Pediatr Endocrinol Rev. 2009; 7:22-31.

12. Boisen KA, Kaleva M, Main KM et al. Difference in prevalence of congenital cryptorchidism in infants between two Nordic countries. Lancet. 2004; 363:1264-9.

13. Hutson JM, Hasthorpe S. Abnormalities of testicular descent. Cell Tissue Res. 2005; 322:155-8.

14. Zivkovic D, Hadziselimovic F. Development of Sertoli cells during mini-puberty in normal and cryptorchid testes. Urol Int. 2009; 82:89-91

15. Bergh A, Söder O. Studies of cryptorchidism in experimental animal models. Acta Paediatr. 2007; 96:617-21.

16. Hadziselimovic F, Zivkovic D, Bica DTG, Emmons LR. The Importance of Mini-Puberty for Fertility in Cryptorchidism. J Urol. 2005; 174:1536-9.

17. Rey RA, Grinspon RP, Gottlieb S et al. Male hypogonadism: an extended classification based on a developmental, endocrine physiology-based approach. Andrology. 2013; 1:3-16.

18. Brioude F, Bouligand J, Trabado S et al. Non-syndromic congenital 
hypogonadotropic hypogonadism: clinical presentation and genotype-phenotype relationships. Eur J Endocrinol. 2010; 162:835-51.

19. Eiholzer U, l'Allemand D, Rousson V et al. Hypothalamic and Gonadal Components of Hypogonadism in Boys with PraderLabhart- Willi Syndrome. J Clin Endocrinol Metab. 2006; 91:892-8.

20. Bergadá I, Andreone L, Bedecarrás P et al. Seminiferous tubule function in delayed-onset X-linked adrenal hypoplasia congenita associated with incomplete hypogonadotrophic hypogonadism. Clin Endocrinol (Oxf). 2008; 68:240-6.

21. Diamond DA, Borer JG, Peters CA et al. Neonatal scrotal haematoma: mimicker of neonatal testicular torsion. BJU Int. 2003; 91:675-7.

22. Cohen LE, Radovick S. Molecular basis of combined pituitary hormone deficiencies. Endocr Rev. 2002; 23:431-42.

23. Silveira LF, MacColl GS, Bouloux PM. Hypogonadotropic hypogonadism. Semin Reprod Med. 2002; 20:327-38.

24. Jennings RW. Prune belly syndrome. Semin Pediatr Surg. 2000; 9:115-20.

25. Ferlin A, Zuccarello D, Zuccarello B et al. Genetic alterations associated with cryptorchidism. JAMA. 2008; 300:2271-6.

26. Gottlieb S, Rey RA, Malozowski S. Klinefelter syndrome and cryptorchidism. JAMA. 2009; 301:1436-7.

27. Bay $\mathrm{K}$, Hartung $\mathrm{S}$, Ivell $\mathrm{R}$ et al. Insulin-like factor 3 serum levels in 135 normal men and 85 men with testicular disorders relationship to the luteinizing hormone-testosterone axis. J Clin Endocrinol Metab. 2005; 90:3410-8.

28. Josso N. Paediatric applications of anti-Müllerian hormone research. Horm Res. 1995; 43:243-8.

29. Lee MM, Donahoe PK, Silverman BL et al. Measurements of serum Müllerian inhibiting substance in the evaluation of children with nonpalpable gonads. N Engl J Med. 1997; 336:1480-6.

30. De Schepper J, Verlinde F, Cortvrindt R et al. Serum inhibin B in normal term-born male and female neonates during the first week of life. Eur J Pediatr. 2000; 159:465-9.

31. Grinspon RP, Ropelato MG, Bedecarrás P et al. Gonadotrophin secretion pattern in anorchid boys from birth to pubertal age: pathophysiological aspects and diagnostic usefulness. Clin Endocrinol (Oxf). 2012; 76:698-705.

32. Bougnères $\mathrm{P}$, François $\mathrm{M}$, Pantalone L et al. Effects of an early postnatal treatment of hypogonadotropic hypogonadism with a continuous subcutaneous infusion of recombinant folliclestimulating hormone and luteinizing hormone. J Clin Endocrinol Metab. 2008; 93:2202-5.

33. Aksglæde L, Petersen JH, Main KM et al. High normal testosterone levels in infants with non-mosaic Klinefelter's syndrome. Eur J Endocrinol. 2007; 157:345-50.

34. Bastida MG, Rey RA, Bergadá I et al. Establishment of testicular endocrine function impairment during childhood and puberty in boys with Klinefelter syndrome. Clin Endocrinol (Oxf). 2007, 67:863-70.

35. Lahlou N, Fennoy I, Carel JC, Roger M. Inhibin B and AntiMullerian Hormone, but not testosterone levels, are normal in infants with nonmosaic Klinefelter Syndrome. J Clin Endocrinol Metab. 2004; 89:1864-8.

36. Ross JL, Samango-Sprouse C, Lahlou N et al. Early androgen deficiency in infants and young boys with 47,XXY Klinefelter syndrome. Horm Res. 2005; 64:39-45.

37. Elder JS. Ultrasonography Is Unnecessary in Evaluating Boys With a Nonpalpable Testis. Pediatrics. 2002; 110:748-51.

38. Nguyen HT, Coakley F, Hricak H. Cryptorchidism: strategies in detection. Eur Radiol. 1999; 9-336.

39. Siemer S, Humke U, Hildebrandt U et al. Diagnosis of nonpalpable testes in childhood: comparison of magnetic resonance imaging and laparoscopy in a prospective study. Eur J Pediatr Surg. 2000 10:114-8.

40. Krishnaswami S, Fonnesbeck C, Penson D, McPheeters ML. Magnetic resonance imaging for locating nonpalpable undescended testicles: a meta-analysis. Pediatrics. 2013; 131:e1908-e16.

41. Ciaccio M, Rivarola MA, Belgorosky A. Decrease of serum sex hormone-binding globulin as a marker of androgen sensitivity. Correlation with clinical response. Acta Endocrinol (Copenh). 1989; 120:540-4.

42. Tapanainen J, Martikainen H, Dunkel L et al. Steroidogenic response to a single injection of hCG in pre- and early pubertal cryptorchid boys. Clin Endocrinol (Oxf). 1983; 18:355-62.

43. Zaccara A, Spagnoli A, Capitanucci ML et al. Impalpable testis and laparoscopy: when the gonad is not visualized. JSLS. 2004; 8:39-42.

44. Grinspon RP, Ropelato MG, Gottlieb S et al. Basal FollicleStimulating Hormone and Peak Gonadotropin Levels after Gonadotropin-Releasing Hormone Infusion Show High Diagnostic Accuracy in Boys with Suspicion of Hypogonadotropic Hypogonadism. J Clin Endocrinol Metab. 2010; 95:2811-8.

45. Wikström AM, Bay K, Hero $\mathrm{M}$ et al. Serum insulin-like factor 3 levels during puberty in healthy boys and boys with Klinefelter syndrome. J Clin Endocrinol Metab. 2006; 91:4705-8.

46. Aksglaede L, Juul A. Testicular function and fertility in men with Klinefelter syndrome: a review. Eur J Endocrinol. 2013; 168:R67-76.

47. Kollin C, Stukenborg JB, Nurmio $M$ et al. Boys with undescended testes: endocrine, volumetric and morphometric studies on testicular function before and after orchidopexy at nine months or three years of age. J Clin Endocrinol Metab. 2012; 97:4588-95.

48. Rey RA. Early orchiopexy to prevent germ cell loss during infancy in congenital cryptorchidism. J Clin Endocrinol Metab. 2012; 97:4358-61.

49. Pettersson A, Richiardi L, Nordenskjöld A et al. Age at surgery for undescended testis and risk of testicular cancer. N Engl J Med. 2007; 356:1835-41

50. Hadziselimovic F. Cryptorchidism, its impact on male fertility. Eur Urol. 2001; 41:121-3.

51. Penson D, Krishnaswami S, Jules A, McPheeters ML. Effectiveness of hormonal and surgical therapies for cryptorchidism: a systematic review. Pediatrics. 2013; 131:e1897-907.

52. Thorsson AV, Christiansen P, Ritzen M. Efficacy and safety of hormonal treatment of cryptorchidism: current state of the art. Acta Paediatr. 2007; 96:628-30

53. Esposito C, Caldamone AA, Settimi A, El-Ghoneimi A Management of boys with nonpalpable undescended testis. Nature Clinical Practice Urology. 2008; 5:252-60.

54. Hack WW, Sijstermans K, van der Voort-Doedens RN. Correction of Cryptorchidism and Testicular Cancer (Letter). N Engl J Med. 2007; 357:826.

55. Myrup C, Schnack TH, Wohlfahrt J. Correction of Cryptorchidism and Testicular Cancer (Letter). N Engl J Med. 2007; 357:825-6.

56. Akre O, Pettersson A, Richiardi L. Risk of contralateral testicula cancer among men with unilaterally undescended testis: A meta analysis. Int J Cancer. 2009; 124:687-9.

57. Sharpe RM, Skakkebæk NE. Testicular dysgenesis syndrome: mechanistic insights and potential new downstream effects. Fertil Steril. 2008; 89:e33-8.

58. Batata MA, Chu FC, Hilaris BS et al. Testicular cancer in cryptorchids. Cancer. 1982; 49:1023-30.

59. Riegler HC. Torsion of intra-abdominal testis: an unusual problem in diagnosis of the acute surgical abdomen. Surg Clin North Am. 1972; 52:371-4

60. Skakkebæk NE, Jørgensen N, Main KM et al. Is human fecundity declining? IntJAndrol. 2006; 29:2-11

61. Hadziselimovic F, Hocht B, Herzog B, Buser MW. Infertility in cryptorchidism is linked to the stage of germ cell development at orchidopexy. Horm Res. 2007; 68:46-52.

62. Hadziselimovic F, Hoecht B. Testicular histology related to fertility outcome and postpubertal hormone status in cryptorchidism. Klin Padiatr. 2008; 220:302-7. 